\title{
Acute Sensory-Motor Axonal Neuropathy in a 57- Year-Old Male Presenting With Paresthesia and Distal Muscle Weakness
}

\author{
Azka Latif $^{1}$, Vikas Kapoor $^{1}$, Akshat Sood ${ }^{1}$, Joseph Thirumalareddy ${ }^{2}$, Abubakar Tauseef ${ }^{1}$ \\ 1. Internal Medicine, CHI Creighton University, Omaha, USA 2. Hospital Medicine, CHI Creighton University, Omaha, \\ USA
}

Corresponding author: Azka Latif, azkalatif@creighton.edu

\begin{abstract}
Guillain-Barré syndrome (GBS) is a relatively uncommon post-infectious, immune-mediated neurologic disorder with an incidence of $0.5-2 / 100,000$. It is usually preceded by an infection that evokes an immune response that cross-reacts with peripheral nerve components via molecular mimicry. The presentation of this disorder has several forms, including acute inflammatory demyelinating polyneuropathy (AIDP), acute motor axonal neuropathy (AMAN), acute motor-sensory axonal neuropathy (AMSAN), and Miller Fisher syndrome (MFS). The case we describe is of a 57-year-old male presenting with sensory features followed by symmetrical ascending paralysis and diagnosed with ASMAN, a recently described subtype of GBS, based on neurological and laboratory findings.
\end{abstract}

Categories: Internal Medicine, Neurology

Keywords: neurologic disorder, paralysis, inflammatory

\section{Introduction}

Guillain-Barré Syndrome (GBS) is a relatively uncommon post-infectious, immune-mediated neurologic disorder with an incidence of $0.5-2 / 100,000$ characterized by the demyelination of spinal nerve roots and peripheral nerves, and presents with a mono-phasic illness with cerebrospinal fluid (CSF) albuminocytological dissociation with partial or complete recovery [1]. GBS is diagnosed on the basis of a clinical evaluation, CSF findings, and the results of nerve conduction studies. In association with supportive care, immunotherapy via intravenous immunoglobulin (IVIG) administration or plasmapheresis, is the standard treatment for GBS [2].

Besides the classic presentation of GBS, clinical variants are based on the types of nerve fibers involved, the predominant mode of fiber injury (demyelinating versus axonal), and the presence of an alteration in consciousness [3]. These variant forms of GBS often do not fulfill existing diagnostic criteria defined for classical GBS [4]. Hadden et al. evaluated the neurophysiology criteria vs recently proposed Rajabally et al. criteria, which classified GBS into a few subcategories, including classical GBS (41,67\%), classic MillerFisher Syndrome (MFS) (6, 10\%), pharyngeal-cervical-brachial (PCB) (3, 5\%), paraparetic GBS (4, 7\%), bifacial weakness with paresthesia (3, 5\%), acute ophthalmoparesis (AO) $(1,2 \%)$, and overlap syndrome (3, $5 \%)$ : one (2\%) with GBS/Bickerstaff brainstem encephalitis overlap and two (3\%) with GBS/MFS overlap [5].

\section{Case Presentation}

A 57-year-old male, with no significant past medical history, presented to the emergency department with the complaint of bilateral lower extremity numbness and tingling for two days that was gradually progressive and associated with bilateral lower limb weakness for one day. He didn't report any history of recent gastrointestinal or respiratory tract infections. There was no history of major surgeries, blood transfusions, or intravenous drug abuse prior to the onset of disease and other comorbid situations.

On clinical examination, the patient was afebrile, conscious, and well-oriented to time, place, and person. The blood pressure was normal (120/75 $\mathrm{mmHg}$ ), pulse rate was normal (82/minute), and respiratory rate was normal (20/minute). On neurological examination, he had an ataxic gait. There was generalized hyporeflexia and decreased power (3/5) in bilateral lower limbs. In view of these clinical findings, a diagnosis of lower motor neuron type paraparesis was made. The initial laboratory workup was unremarkable. Magnetic resonance imaging (MRI) was obtained to rule out a cerebrovascular accident, which did not reveal any acute changes. While being in the hospital, his lower extremity weakness gradually worsened, followed by numbness and tingling sensation in the bilateral upper extremity.

Further workup with CSF is shown in Table 1. 


\section{Cureus}

\begin{tabular}{|l|l|}
\hline Appearance & Clear \\
\hline Color & Colorless \\
RBCs & $15 / \mathrm{uL}$ \\
TLC & $2 / \mathrm{uL}$ \\
Glucose & $65 \mathrm{mg} / \mathrm{dl}$ \\
Proteins & $200 \mathrm{mg} / \mathrm{dll}$ \\
\hline
\end{tabular}

TABLE 1: CSF analysis

CSF: cerebrospinal fluid

Albuminocytologic dissociation raised concern for GBS, therefore, he was started on intravenous immune globulins at a dose of $0.4 \mathrm{~g} / \mathrm{kg}$ per day for a total of five days. Supportive measures, such as the administration of intravenous fluids and nutritional therapy, were also started. Over the course of hospital stay, his neurologic symptoms gradually started to improve. He worked with a physical and occupational therapist and was discharged in two months. On discharge, he was able to walk with assistance.

\section{Discussion}

All patients initially identified as GBS or related disorders can be subclassified as having classical GBS, classic Miller-Fisher Syndrome (MFS), ASMAN, pharyngeal-cervical-brachial (PCB), paraparetic GBS, bifacial weakness with paresthesia, acute ophthalmoparesis (AO), and overlap syndrome [1]. Pure sensory GBS is a very rare sub-type; to date, $<10$ cases of pure sensory GBS have been reported; thus, the clinical and pathological features of sensory variant GBS are yet to be well-characterized [6].

ASMAN is a recently described subtype of GBS characterized by the acute onset of sensory symptoms, with a loss of deep tendon reflexes and distal weakness. Electrophysiological studies show mildly reduced nerve conduction velocities in axons combined with a marked reduction of muscle action and sensory nerve action potentials.

Several points of this case are remarkable. Patients with AMSAN, like the case described above, usually experience severe symptoms over a short period of time. They also have a prolonged and incomplete phase of recovery despite treatment with immunomodulatory drugs [7]. The therapy of AMAN and AMSAN is based on that of the acute inflammatory demyelinating polyradiculoneuropathy (AIDP), that is, plasma exchange and intravenous immunoglobulin (IVIG) while the benefit of steroids remains ambiguous [8].

\section{Conclusions}

To conclude, in ASMAN, immunomodulating therapy with IVIG may lead to full recovery in selected cases. Although rare, inflammatory polyradiculoneuropathy should be considered in the differential diagnosis of acute flaccid quadriparesis with length-dependent sensory dysfunction.

\section{Additional Information \\ Disclosures}

Human subjects: Consent was obtained by all participants in this study. Conflicts of interest: In compliance with the ICMJE uniform disclosure form, all authors declare the following: Payment/services info: All authors have declared that no financial support was received from any organization for the submitted work. Financial relationships: All authors have declared that they have no financial relationships at present or within the previous three years with any organizations that might have an interest in the submitted work. Other relationships: All authors have declared that there are no other relationships or activities that could appear to have influenced the submitted work.

\section{References}

1. Yuki N, Hartung H: Guillain-Barre syndrome. N Engl J Med. 2012, 3:2294-2304. 10.1056/NEJMra1114525

2. Meena AK, Khadilkar SV, Murthy JMK: Treatment guidelines for Guillain-Barré syndrome. Ann Indian Acad Neurol. 2011, 14:S73-S81. 10.4103/0972-2327.83087

3. Fisher M: An unusual variant of acute idiopathic polyneuritis (syndrome of ophthalmoplegia, ataxia and areflexia). N Engl J Med. 1956, 2:57-65. 10.1056/NEJM195607122550201

4. Asbury AK, Cornblath DR: Assessment of current diagnostic criteria for Guillain-Barre syndrome . Ann Neurol. 1990, 27:S21-24. 


\section{Cureus}

5. Hiew FL, Ramlan R, Viswanathan S, Puvanarajah S: Guillain-Barré syndrome, variants \& forms fruste: reclassification with new criteria. Clin Neurol Neurosurg. 2017, 158:114-118. 10.1016/j.clineuro.2017.05.006

6. Yang J, Haun M, Jiang H, Song C, Zhong L, Liang Z: Pure sensory Guillain-Barré syndrome: a case report and review of the literature. Nat Rev Neurol. 2014, 08:1397-1401. 10.3892/etm.2014.1955

7. Rostásy KM, Huppke P, Beckers B, et al.: Acute motor and sensory axonal neuropathy (AMSAN) in a 15year-old boy presenting with severe pain and distal muscle weakness. Neuropediatrics. 2005, 36:260-264 10.1055/s-2005-865774

8. Chowdhury D, Arora A: Axonal Guillain-Barré syndrome: a critical review . Acta Neurol Scand. 2001, 103:260-264. 